\title{
Nonequilibrium Model of Photon Condensation
}

\author{
Peter Kirton and Jonathan Keeling \\ SUPA, School of Physics and Astronomy, University of St Andrews, St Andrews KY16 9SS, United Kingdom
} (Received 10 April 2013; published 4 September 2013)

\begin{abstract}
We develop a nonequilibrium model of condensation and lasing of photons in a dye filled microcavity. We examine in detail the nature of the thermalization process induced by absorption and emission of photons by the dye molecules, and investigate when the photons are able to reach a thermal equilibrium Bose-Einstein distribution. At low temperatures, or large cavity losses, the absorption and emission rates are too small to allow the photons to reach thermal equilibrium and the behavior becomes more like that of a conventional laser.
\end{abstract}

DOI: 10.1103/PhysRevLett.111.100404

Bose-Einstein condensation (BEC) has been observed in a wide variety of systems, from ultracold atomic gases $[1,2]$ to quasiparticles in solid state systems such as polaritons [3-6], excitons [7], and magnons [8]. Recently experiments have shown convincing evidence of a BoseEinstein distribution, and macroscopic occupation of the lowest mode for a gas of photons confined in a dye-filled optical microcavity [9-12]. In these experiments, the thermal equilibrium distribution of photons arises because of phonon dressing of the absorption and emission by the dye molecules, and the rapid thermalization of rovibrational modes of the dye molecules by their collisions with the solvent. This leads to the accumulation of low-energy photons, closely following a Bose-Einstein distribution, as is clearly seen experimentally [10].

Such a system is very closely related to a dye laser [13], but differs in the near-thermal emission spectrum that is observed below and near the threshold density and in the fact that the macroscopic population occurs at the minimum energy mode of the cavity and is not related to the gain maximum of the dye [10]. There are also close connections to microlasers [14]. However microlasers, having strong coupling between the gain medium and cavity, display thresholdless lasing [15]. In contrast, the observed behavior in the photon condensate [10] is that there is a sharp threshold which occurs far below inversion.

In the context of polariton condensation [3-6] there has been much debate $[16,17]$ about the extent to which the lack of true thermal equilibrium in experiments means the system should be called a condensate or a laser. However, various calculations for polaritons, from quantum kinetics $[18,19]$ to Schwinger-Keldysh path integrals [20], have found a relatively smooth crossover between behavior typical of a laser, and that typical of an equilibrium condensate. Both lasers and condensates involve a spontaneous phase-symmetry breaking, and a transition to a macroscopically occupied mode, and so their connection has long been recognized [21]. The photon condensate system provides a further example of a system in which the distinction between Bose condensation and lasing must be carefully examined.
PACS numbers: 03.75.Hh, 42.55.Mv, 67.85.Hj, 71.38.-k

The nature of the thermalization process in the photon condensate differs significantly from that found in other systems which exhibit BEC. There are no direct photonphoton interactions in the cavity and the thermal BoseEinstein distribution seen in this system can be understood as arising from the combination of asymmetry between absorption and emission (the Kennard-Stepanov relation [22]) and the retrapping of fluorescence. This mechanism and the presence of dissipation (loss) raises similar questions to those raised for polaritons: Can the observed behavior be understood as an exotic form of lasing? What features distinguish a Bose-Einstein condensate from an exotic laser? To address these questions, we show that, starting from a model of stimulated emission, i.e., that of a modified laser, we can describe the observed Bose-Einstein distribution of light. For experimental parameters (low losses), we find that the above threshold state is practically indistinguishable from the "textbook" [23] condensate of a noninteracting Bose gas. Significant deviations from the thermal behavior occur if the cavity losses increase, and a crossover toward more standard lasing is observed. Our results therefore show that, even in an open system, stimulated emission can produce a momentum distribution indistinguishable from that arising in thermodynamic equilibrium.

Previous theoretical work has attempted to produce models of this system from the point of view of equilibrium statistical mechanics $[24,25]$, while other work has examined the emergence of phase coherence in a BEC where particles interact through an intermediate medium [26]. We aim instead to provide a general nonequilibrium framework for understanding the steady state properties of the photons, taking into account the pump and decay processes. This allows one to understand how these compete with the thermalization process, and control when the system behaves like a laser or like a condensate.

A schematic diagram of our model is shown in Fig. 1. This consists of photon modes, labeled $m$, with creation operators $a_{m}^{\dagger}$, confined in the optical cavity coupled to a single electronic transition of the dye molecules. Each dye molecule, indexed by the label $i$, is represented as a 


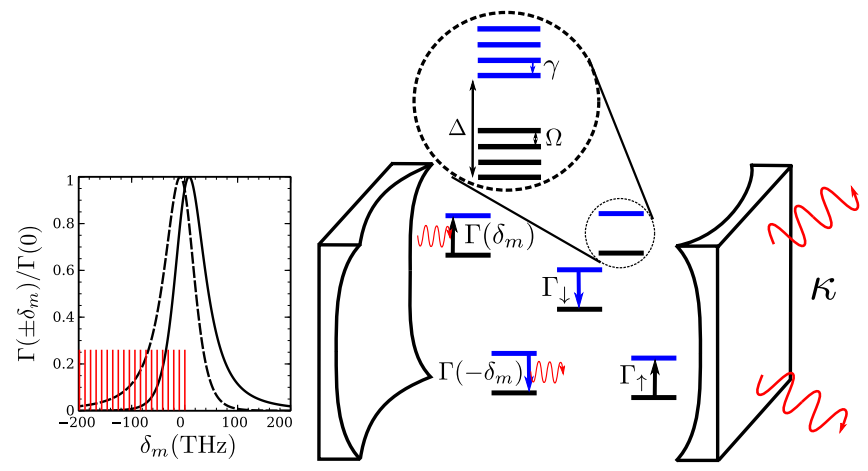

FIG. 1 (color online). Cartoon of the system showing the decay processes included in Eq. (2). The zoomed in view shows the energy level structure of the dye molecules. The graph shows the characteristic behavior of the emission rate, $\Gamma\left(-\delta_{m}\right)$ (dashed line) and the absorption rate, $\Gamma\left(\delta_{m}\right)$ (solid line) described in the main text. The vertical (red) lines show the typical spacing of the photon modes confined in the cavity.

two-level system, with the Pauli matrix $\sigma_{i}$ and splitting $\Delta$ between ground and excited levels. These levels are dressed by ladders of rovibrational states, which can be thought of as an on-site phonon [13], described by operators $b_{i}, b_{i}^{\dagger}$. The level scheme is shown in Fig. 1 . The Hamiltonian is thus

$$
\begin{aligned}
H= & \sum_{m} \omega_{m} a_{m}^{\dagger} a_{m}+\sum_{i} \frac{\Delta}{2} \sigma_{i}^{z}+\Omega\left(b_{i}^{\dagger} b_{i}+\sqrt{S} \sigma_{i}^{z}\left(b_{i}+b_{i}^{\dagger}\right)\right) \\
& +g \sum_{m, i}\left(a_{m} \sigma_{i}^{+}+a_{m}^{\dagger} \sigma_{i}^{-}\right)
\end{aligned}
$$

using units such that $\hbar=k_{B}=1$. As in the experiment [10], we consider photon modes in a two-dimensional harmonic potential (arising from the curvature of the mirrors). We therefore take regularly spaced oscillator levels $\omega_{m}=$ $\omega_{0}+m \epsilon$, having a degeneracy $g_{m}$ given by $g_{m}=m+1$. The lowest frequency $\omega_{0}$ is the "cavity cutoff." If in equilibrium, condensation would lead to a macroscopic occupation of this mode. In the following we quote frequencies relative to the molecular splitting $\Delta$ and thus introduce $\delta_{m}=\omega_{m}-\Delta$. Since the light-matter coupling is small compared to optical frequencies, we assume a JaynesCummings coupling, with frequency independent coupling strength $g$. The vibrational mode spacing is $\Omega$, and the interaction between electronic and vibrational states is given by the Huang-Rhys parameter $S$, which characterizes the difference in phonon displacement between the ground and excited states. The parameter values we use, corresponding to the experiment [10], are given in the figure captions.

To model the open system, we must add additional loss processes and external pumping. We include the loss of cavity photons with rate $\kappa$, assumed independent of the photon frequency, and a rate $\Gamma_{\downarrow}$ describing fluorescence of the dye molecules into noncavity modes. To balance these losses we include pumping with rate $\Gamma_{\uparrow}$. These processes may all be described by standard Markovian Lindblad terms, as there is no significant thermal occupation of relevant photon modes outside the cavity. The localized vibrational modes also undergo incoherent relaxation, due to scattering off of solvent molecules. This is modeled as a relaxation rate $\gamma$ toward a thermal equilibrium state at temperature $T$. These processes cannot be described by Markovian loss rates, as this cannot describe thermalization of the radiation $[27,28]$. Below we describe an alternate approach to include these processes.

If the coupling to phonons $S$ is reasonably strong, then multiphonon effects will be important in describing the thermalization processes. These can be captured by making a polaron transformation $H \rightarrow U^{\dagger} H U$, where $U=$ $\exp \left[\sum_{i} \sqrt{S} \sigma_{i}^{z}\left(b_{i}-b_{i}^{\dagger}\right)\right]$. Since the coupling of molecules to the optical modes is weak, we then treat the dynamics perturbatively in $g$ while keeping all orders of $S$. Working in the interaction picture, and expanding the Liouville equation to second order in $g$, one may then trace out the degrees of freedom associated with the vibrational mode and its damping. The resulting equation of motion then contains Lindblad terms which cause simultaneous transitions in both the photon field and the dressed molecule [29]. These processes then describe the emission (absorption) of photons into (from) the cavity, as shown schematically in Fig. 1. Including all processes, the resulting master equation for the photon-molecule system is

$$
\begin{aligned}
\dot{\rho}= & -i\left[H_{0}, \rho\right]-\sum_{i, m}\left\{\frac{\kappa}{2} \mathcal{L}\left[a_{m}\right]+\frac{\Gamma_{\uparrow}}{2} \mathcal{L}\left[\sigma_{i}^{+}\right]+\frac{\Gamma_{\downarrow}}{2} \mathcal{L}\left[\sigma_{i}^{-}\right]\right. \\
& \left.+\frac{\Gamma\left(-\delta_{m}\right)}{2} \mathcal{L}\left[a_{m}^{\dagger} \sigma_{i}^{-}\right]+\frac{\Gamma\left(\delta_{m}\right)}{2} \mathcal{L}\left[a_{m} \sigma_{i}^{+}\right]\right\} \rho .
\end{aligned}
$$

Here, the system Hamiltonian is $H_{0}=\sum_{m, i} \tilde{\delta}_{m} a_{m}^{\dagger} a_{m}+$ $\eta_{m} a_{m}^{\dagger} a_{m} \sigma_{i}^{+} \sigma_{i}^{-}$and $\mathcal{L}[X] \rho=\left\{X^{\dagger} X, \rho\right\}-2 X \rho X^{\dagger}$ is the usual Lindblad term. The phonon assisted emission and absorption rates, along with the Lamb shifts, are related to the function [29]

$$
K(\omega)=g^{2} \int_{0}^{\infty} d t f(t) \mathrm{e}^{-\left(\Gamma_{\uparrow}+\Gamma_{\downarrow}\right)|t| / 2} \mathrm{e}^{-i \omega t},
$$

where $f(t)$ is a correlation function of polaron operators [29-31] given by

$f(t)=\exp \left[-\frac{2 S \gamma}{\pi} \int_{-\infty}^{\infty} d \nu \frac{2 \sin ^{2} \frac{\nu t}{2} \operatorname{coth} \frac{\beta \nu}{2}+i \sin \nu t}{(\Omega-\nu)^{2}+\frac{\gamma^{2}}{4}}\right]$.

The absorption and emission rates in the master equation are simply given by $\Gamma(\omega)=2 \operatorname{Re}[K(\omega)]$ while the energy shifts in the Hamiltonian are $\eta_{m}=\operatorname{Im}\left[K\left(-\delta_{m}\right)-K\left(\delta_{m}\right)\right]$ and $\tilde{\delta}_{m}=\delta_{m}+\operatorname{Im}\left[K\left(\delta_{m}\right)\right]$. These Lamb shifts do not affect the populations at order $g^{2}$; truncating at this order is valid below threshold in weak coupling. In the following we focus on the behavior below and at threshold and thus 
set $\eta_{m}=0$ and $\tilde{\delta}_{m}=\delta_{m}$ [29]. An illustration of the decay rates, as a function of detuning, is shown in Fig. 1. We note that for frequencies where the rates $\Gamma_{\uparrow}, \Gamma_{\downarrow}$ can be ignored in Eq. (3), the vibration induced emission and absorption rates are related by a Boltzmann factor $\Gamma(\delta)=\mathrm{e}^{\beta \delta} \Gamma(-\delta)$ [32] with $\beta$ corresponding to the phonon (solvent) temperature, thus satisfying the Kennard-Stepanov relation between absorption and emission [22]. At large frequencies $\Gamma(\omega)$ ceases to obey this relation because the incoherent pumping process corresponds to coupling to a white noise (i.e., infinite temperature) bath [28].

Marthaler et al. [29] considered this kind of master equation as a route to lasing without inversion in circuit QED. The same mechanism they proposed also applies for the photon condensate, allowing coherent emission far below inversion: If $\Gamma\left(-\delta_{m}\right)>\Gamma\left(\delta_{m}\right)$ then the asymmetry in emission and absorption induced by thermalization with the phonons allows net gain without inversion. For lasing to occur significantly below the inversion point we require $\delta_{0} \ll-T$ so that the asymmetry of the absorption and emission rates is sufficiently large. As we will discuss below, the same conditions lead to thermalized lasing, as long as the relevant emission rates $\Gamma\left(-\delta_{m}\right)$ are large enough to overcome the losses from the cavity.

We can use the master equation, Eq. (2), to derive a semiclassical rate equation for the population of each photon mode which, after adiabatically eliminating the $N$ molecular degrees of freedom, is

$\frac{\partial n_{m}}{\partial t}=-\kappa n_{m}+N \frac{\Gamma\left(-\delta_{m}\right)\left(n_{m}+1\right) \tilde{\Gamma}_{\uparrow}-\Gamma\left(\delta_{m}\right) n_{m} \tilde{\Gamma}_{\downarrow}}{\tilde{\Gamma}_{\uparrow}+\tilde{\Gamma}_{\downarrow}}$,

where we define $\tilde{\Gamma}_{\uparrow}=\Gamma_{\uparrow}+\sum_{m} g_{m} \Gamma\left(\delta_{m}\right) n_{m}$ and $\tilde{\Gamma}_{\downarrow}=\Gamma_{\downarrow}+$ $\sum_{m} g_{m} \Gamma\left(-\delta_{m}\right)\left(n_{m}+1\right)$. We can then use the steady state of this expression in combination with the rates from Eq. (3) to calculate the photon population in each mode, $g_{m} n_{m}$. Note that the $n_{m}+1$ term in the emission process corresponds to the trapping of spontaneous fluorescence from the dye. When combined with the Kennard-Stepanov relation discussed above, this means that in the equilibrium limit, $\kappa, \Gamma_{\uparrow}, \Gamma_{\downarrow} \rightarrow 0$, the stationary solution to this equation is an equilibrium Bose-Einstein distribution. i.e. $\left(n_{m}+1\right) /$ $n_{m}=e^{\beta \delta_{m}} \tilde{\Gamma}_{\downarrow} / \tilde{\Gamma}_{\uparrow}$. Far below threshold, where $n_{m} \ll 1$, this corresponds to an effective chemical potential $\mu_{\text {eff }}=T \ln \left(\Gamma_{\uparrow} / \Gamma_{\downarrow}\right)$. When occupation is not negligible, the dependence of $\tilde{\Gamma}_{\downarrow}$ and $\tilde{\Gamma}_{\uparrow}$ on $n_{m}$ implies a self-consistency condition on $\mu$, so that $\mu \rightarrow \delta_{0}$ as pumping increases. This equilibrium limit is discussed in Ref. [24]. Applying our rate equations above threshold the model continues to predict noninteracting BEC behavior; i.e., photons accumulate in the lowest energy mode and the thermal tail saturates. This is in contrast to the experiment [10] where the population in the tail continues to grow as expected for a trapped, interacting BEC [34]. However, as noted above, beyond threshold terms of order $g^{4}$ and above must be
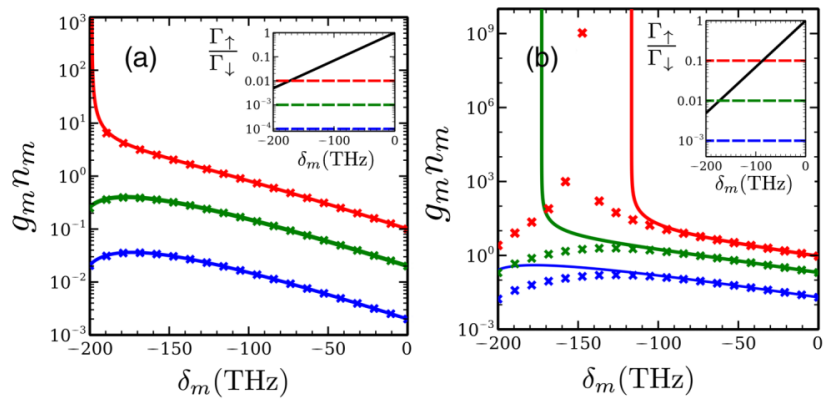

FIG. 2 (color online). Mode populations $g_{m} n_{m}$ vs detuning $\delta_{m}$ for various pump strengths. Crosses are results of the nonequilibrium model, and lines show Bose-Einstein distributions fitted to the tail of the numerical results. Insets show the pump powers, $\Gamma_{\uparrow} / \Gamma_{\downarrow}=\mathrm{e}^{\beta \mu_{\text {eff }}}$, (dashed lines) compared to the ratio $\Gamma\left(-\delta_{m}\right) / \Gamma\left(\delta_{m}\right) \simeq \mathrm{e}^{\beta \delta_{m}}$ (solid, black line) which gives a good approximation to the threshold. Panel (a) corresponds to experimental losses, $\kappa=10 \mathrm{MHz}$. Panel (b) shows $\kappa=5 \mathrm{GHz}$ where losses prevent thermalization. Other parameters are: $\gamma=100 \mathrm{THz}$, $\Gamma_{\downarrow}=1 \mathrm{GHz}, S=0.5, \Omega=1 \mathrm{THz}, N=10^{11}, g=0.1 \mathrm{GHz}, T=$ $300 \mathrm{~K}, \delta_{0}=-200 \mathrm{THz}$, and the mode spacing $\epsilon=10 \mathrm{THz}$.

retained, describing thermalization of a BEC in which the interactions are mediated by scattering [34,35].

In Fig. 2(a) we present results including losses for parameter values (given in the caption) typical of those in the experiments of Refs. [9-11]. We fit Bose-Einstein distributions to the data by tuning the chemical potential so that the tail matches the numerical results. These distributions agree very closely with the numerical results, even in the presence of losses. The profile changes dramatically when the cavity loss rate is increased, as shown in Fig. 2(b). In this case the losses for the lowest energy modes exceeds the gain, controlled by the rate $\Gamma\left(-\delta_{0}\right)$, thus preventing these modes from reaching thermal equilibrium. As can be clearly seen in the figure, the modes with higher energy (i.e., $\delta_{m} \simeq 0$ ) still match the BoseEinstein distributions well. At sufficiently strong pumping there is a threshold (at much higher power than would be required if in thermal equilibrium) above which we find a macroscopic peak appears in an excited mode of the cavity. This mode is determined by the lowest mode such that $\Gamma\left(-\delta_{m}\right)$ is large enough to overcome the losses. For the parameters of Fig. 2(b) the thermalization still plays a role in the rates of emission and absorption, so that it is not the mode with peak emission rate (near $\delta_{m}=0$ ) which becomes macroscopically occupied. However, at yet higher decay rates, the behavior crosses over toward such "standard" laser behavior, and all thermal properties are lost. Similarly, as $T \rightarrow 0$ the emission and absorption spectra become a narrow Lorentzian peak centered at $\delta_{m}=0$, and the lasing mode moves to the center of the gain peak.

Since the origin of the destruction of thermalization is the competition between loss $\kappa$ and emission rate $\Gamma\left(-\delta_{0}\right)$, it is also clear that lowering the cavity cutoff (making $\delta_{0}$ 
more negative) has a similar effect to increasing the losses. Equilibrium behavior can only be seen when the cavity cutoff is sufficiently close to the molecular frequency, ensuring that $\Gamma\left(-\delta_{0}\right)$ is sufficiently large.

In order to explore the degree of thermalization as a function of temperature and loss rates, we next consider the behavior at threshold. We examine two different aspects: the threshold pump power (a measure typical when considering lasing), and the total photon density at threshold (a measure typical for an equilibrium condensation transition). Figure 3(a) shows the total number of photons in the cavity, $N_{\text {tot }}=\sum_{m} g_{m} n_{m}$, as a function of the pump rate, $\Gamma_{\uparrow}$, at various temperatures. As expected, increasing the temperature of the phonons reduces the asymmetry between $\Gamma\left(\delta_{m}\right)$ and $\Gamma\left(-\delta_{m}\right)$, and thus both the pump power and total density at threshold increase. To explore temperature dependence we identify the threshold as the lowest pump power where $\max \left(n_{m}\right)>T / \epsilon$, with $\epsilon$ the mode spacing - note that this maximally occupied mode is not necessarily the lowest energy mode [36].

Figure 3(b) shows the threshold pump power vs temperature at various cavity loss rates. As $T$ decreases the absorption and emission rates at a given detuning $\delta_{m}$ decrease. At high temperatures and small losses $\kappa$ we see very good agreement with the equilibrium prediction
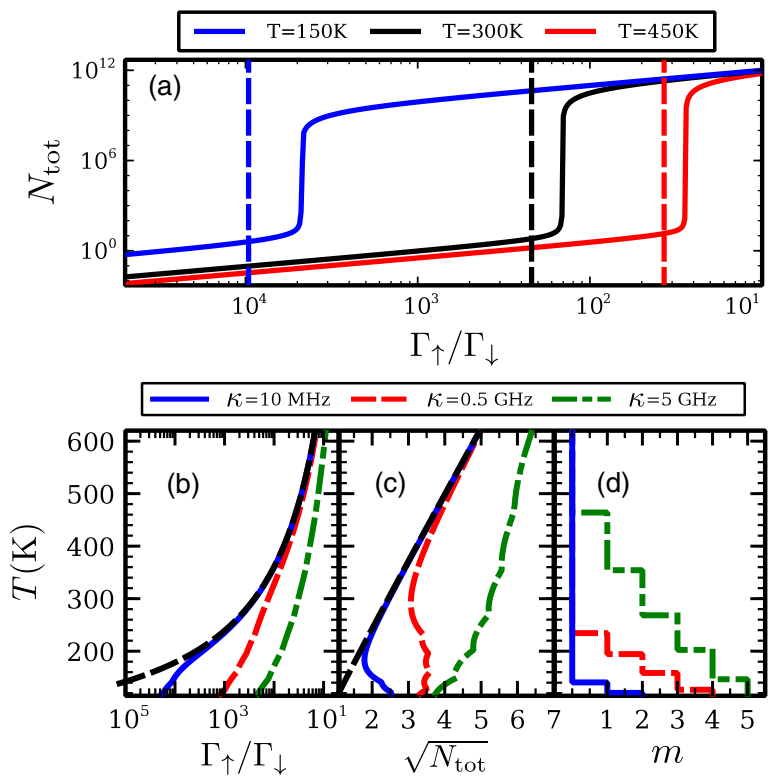

FIG. 3 (color online). (a) Total number of photons in the cavity $N_{\text {tot }}$ vs pump power. The dashed vertical lines show the threshold [defined by $\max \left(n_{m}\right)=T / \epsilon$ ]. (b) Threshold pump power vs temperature, for various cavity loss rates as indicated. The dashed (black) lines show the same threshold calculated for the equilibrium theory. (c) Population $N_{\text {tot }}$ at threshold vs temperature for the same loss rates shown in (b). The dashed (black) line is the equilibrium prediction of critical density. (d) Index of the mode which gains a macroscopic occupation for the same loss rates as in (b). All other parameters are the same as in Fig. 2.
$\Gamma_{\uparrow} / \Gamma_{\downarrow}=\mathrm{e}^{\beta \delta_{0}} /(1+\beta \epsilon)$. At low temperatures the loss rates exceed the gain for the lowest frequencies, and thermalization breaks down. The temperature at which this happens increases with increasing $\kappa$. For the strongly lossy case, shown by the dot-dashed (green) curve [corresponding to the $\kappa$ used in Fig. 2(b)] the threshold remains significantly higher than the equilibrium limit across the whole temperature range shown. In the high temperature limit the emission and absorption rates are symmetric, so the threshold pump strength eventually becomes that required to reach inversion.

A more common description of the "threshold" for equilibrium condensation is the temperature dependent critical number of particles, i.e., the total number of photons in the cavity at threshold. For particles confined in 2D one expects $N_{\text {tot }}=\pi^{2} T^{2} / 6 \epsilon^{2}$ [23]. In Fig. 3(c) we plot this critical number for the same values of $\kappa$ as in Fig. 3(b). Alongside these results we also plot, as the dashed (black) curve, the equilibrium result as above.

For small cavity losses we see that, for temperatures above $\sim 200 \mathrm{~K}$, the agreement between the equilibrium results and the numerics is very close. Below this temperature, the mode which gains a macroscopic occupation is no longer the lowest energy mode, and the system requires stronger pumping to go past the threshold. This leads to an increase in the critical number of photons at low temperatures. As $\kappa$ is increased we again see that the temperature above which the results match a Bose distribution increases. There are also notable kinks in the critical number at low temperatures. These occur as the mode which gains a macroscopic population jumps to higher and higher energy. To illustrate this we show in Fig. 3(d) how the index of the mode with the largest occupation at threshold varies with temperature. We see clearly that the kinks in the photon number correspond to jumps in the maximally occupied mode and that the regions which agree with the equilibrium theory only occur when the ground state has the largest occupation.

In conclusion, we have presented a simple nonequilibrium model which accurately describes the steady state properties of the dye filled cavity systems used to observe condensation of photons. We found that, for relevant parameters, our model accurately predicts the transition to a condensed phase and the equilibrium dependence of pump power and critical photon number on temperature. If the losses from the cavity are increased, the temperature reduced, or the detuning increased compared to those used in the experiment, then a crossover occurs toward behavior more typical of a laser, and thermalization is suppressed. These results show that, as for polariton condensation [20], a smooth crossover between typical laser behavior and equilibrium condensation can arise. Future studies of the time dynamics of how coherence arises, and the thermal distribution emerges following the switch-on of the pump can help to clarify this behavior, and can be predicted using the model presented here. 
The authors would like to thank M. Weitz and J. Klaers for useful discussions and acknowledge financial support from EPSRC program “TOPNES" (EP/I031014/1) and EPSRC (EP/G004714/2).

[1] M.H. Anderson, J.R. Ensher, M.R. Matthews, C.E. Wieman, and E. A. Cornell, Science 269, 198 (1995).

[2] K. B. Davis, M.-O. Mewes, M. R. Andrews, N. J. van Druten, D. S. Durfee, D. M. Kurn, and W. Ketterle, Phys. Rev. Lett. 75, 3969 (1995).

[3] J. Kasprzak, M. Richard, S. Kundermann, A. Baas, P. Jeambrun, J. M. J. Keeling, F. M. Marchetti, M. H. Szymaska, R. André, J. L. Staehli, V. Savona, P. B. Littlewood, B. Deveaud, and L.S. Dang, Nature (London) 443, 409 (2006).

[4] R. Balili, V. Hartwell, D. Snoke, L. Pfeiffer, and K. West, Science 316, 1007 (2007).

[5] H. Deng, H. Haug, and Y. Yamamoto, Rev. Mod. Phys. 82, 1489 (2010).

[6] I. Carusotto and C. Ciuti, Rev. Mod. Phys. 85, 299 (2013).

[7] A. A. High, J. R. Leonard, A. T. Hammack, M. M. Fogler, L. V. Butov, A. V. Kavokin, K. L. Campman, and A. Gossard, Nature (London) 483, 584 (2012).

[8] S. O. Demokritov, V.E. Demidov, O. Dzyapko, G. A. Melkov, A. A. Serga, B. Hillebrands, and A. N. Slavin, Nature (London) 443, 430 (2006).

[9] J. Klaers, F. Vewinger, and M. Weitz, Nat. Phys. 6, 512 (2010).

[10] J. Klaers, J. Schmitt, F. Vewinger, and M. Weitz, Nature (London) 468, 545 (2010).

[11] J. Klaers, J. Schmitt, T. Damm, F. Vewinger, and M. Weitz, Appl. Phys. B 105, 17 (2011).

[12] J. Schmitt, T. Damm, F. Vewinger, M. Weitz, and J. Klaers, New J. Phys. 14, 075019 (2012).

[13] Dye Lasers, edited by F. P. Schäfer (Springer-Verlag, New York, 1990), 3rd ed.

[14] H. Walther, B. T. H. Varcoe, B.-G. Englert, and T. Becker, Rep. Prog. Phys. 69, 1325 (2006).

[15] P. R. Rice and H.J. Carmichael, Phys. Rev. A 50, 4318 (1994).

[16] L. V. Butov, Nature (London) 447, 540 (2007).

[17] D. Snoke, Nat. Phys. 4, 673 (2008).
[18] T. D. Doan, H. T. Cao, D. B. Tran Thoai, and H. Haug, Phys. Rev. B 72, 85301 (2005); 74, 115316 (2006); 78, 205306 (2008).

[19] J. Kasprzak, D. D. Solnyshkov, R. André, L. S. Dang, and G. Malpuech, Phys. Rev. Lett. 101, 146404 (2008).

[20] M. H. Szymanska, J. Keeling, and P. B. Littlewood, Phys. Rev. Lett. 96, 230602 (2006); in Quantum Gases: Finite Temperature and Non-Equilibrium Dynamics, edited by N.P. Proukakis, S. Gardiner, M. J. Davis, and M. H. Szymanska (Imperial College Press, London, 2013).

[21] H. Haken, Rev. Mod. Phys. 47, 67 (1975).

[22] E. H. Kennard, Phys. Rev. 11, 29 (1918); Phys. Rev. 28, 672 (1926); B. I. Stepanov, Dokl. Akad. Nauk SSSR 112, 839 (1957).

[23] L.P. Pitaevskii and S. Stringari, Bose-Einstein Condensation (Clarendon Press, Oxford, 2003).

[24] J. Klaers, J. Schmitt, T. Damm, F. Vewinger, and M. Weitz, Phys. Rev. Lett. 108, 160403 (2012).

[25] D. N. Sob'yanin, Phys. Rev. E 85, 061120 (2012).

[26] D. W. Snoke and S. M. Girvin, J. Low Temp. Phys. 171, 1 (2013).

[27] G. W. Ford and R. F. O’Connell, Phys. Rev. Lett. 77, 798 (1996).

[28] M. Lax, Opt. Commun. 179, 463 (2000).

[29] M. Marthaler, Y. Utsumi, D. S. Golubev, A. Shnirman, and G. Schön, Phys. Rev. Lett. 107, 093901 (2011).

[30] I. Wilson-Rae and A. Imamoglu, Phys. Rev. B 65, 235311 (2002).

[31] D.P.S. McCutcheon and A. Nazir, Phys. Rev. B 83, 165101 (2011).

[32] This expression arises due to a Kubo-Martin-Schwinger relation [33] between the time domain rates $f(t)=$ $f(-t-i \beta)$.

[33] R. Kubo, J. Phys. Soc. Jpn. 12, 570 (1957); P. Martin and J. Schwinger, Phys. Rev. 115, 1342 (1959).

[34] N. Tammuz, R. P. Smith, R. L. D. Campbell, S. Beattie, S. Moulder, J. Dalibard, and Z. Hadzibabic, Phys. Rev. Lett. 106, 230401 (2011).

[35] M. Wouters, Phys. Rev. B 85, 165303 (2012).

[36] This definition corresponds to expanding around the equilibrium thermodynamic limit $[37,38]$ to next-to-leadingorder in the small parameter $\epsilon / T$.

[37] V. Bagnato and D. Kleppner, Phys. Rev. A 44, 7439 (1991).

[38] Z. Hadzibabic, P. Krüger, M. Cheneau, S. P. Rath, and J. Dalibard, New J. Phys. 10, 045006 (2008). 